\title{
Measurement of Alveolar Recruitment at the Bedside: The Beginning of a New Era in Respiratory Monitoring?
}

In this issue of Respiratory Care, Wallet and coworkers bring convincing evidence that PEEP-induced alveolar recruitment can be inferred from regional changes in lung densities computed on frontal bedside digital chest radiographies obtained at 2 different PEEP levels in patients with ARDS. ${ }^{1}$ The reference method for the comparison was the pressure-volume (PV) curve method, one of the methods commercially available at the bedside for measuring PEEP-induced alveolar recruitment. This original finding renews the place of bedside chest radiographies that are routinely performed in critically ill patients with ARDS: they could serve not only as a qualitative tool for assessing lung aeration but also as a monitoring tool for quantifying alveolar recruitment.

See the Original Study on Page 416

As good scientists, the authors point out some serious limitations of the technique that make clinical applicability difficult. Technical conditions as well as the patient's position should be strictly identical between each radiograph. The radiologist should manually delineate 7 polygonal shaped regions of interest in posterior intercostal spaces, on which mean density changes between each PEEP level should be measured using Digital Imaging and Communication in Medicine software. A 5-second end-inspiratory pause is necessary to take the chest radiograph, requiring an anesthetized and paralyzed patient. Due to the lack of calibration in density measurement, only ratios of density between each PEEP can be calculated. A ratio below 1 indicates lung recruitment, and a ratio $\geq 1$ indicates no recruitment or derecruitment. Last but not least, a decrease in density ratio in non-dependent parts of the chest wall may be caused by tidal hyperinflation rather than recruitment. Therefore, the method may require a long time before it can be used routinely at the bedside, justifying the development of alternative options. ${ }^{2-4}$

More than 20 years ago, Ranieri et al showed that PEEPinduced lung recruitment could be assessed by performing static PV curves and measuring end-expiratory lung volume corresponding to different PEEP levels. ${ }^{5}$ Use of PV curves for measuring recruitment in patients with ARDS relies on a strong rationale. In patients with healthy lungs fully aerated at end-expiration, static PV curves depend exclusively on the elastance/compliance of lung parenchyma. In ARDS patients without normally aerated lung regions at zero PEEP (diffuse ARDS characterized by diffuse radiological attenuations resulting in the well-known aspect of "white lung"), static PV curves can be considered as recruitment curves, ${ }^{6}$ and lung recruitment is equal to the increase in lung volume resulting from the increase in intrathoracic pressure. The first milliliters penetrating into the respiratory system at the beginning of the inflation maneuver are entering poorly aerated lung regions and contribute to their recruitment. ${ }^{2,7-9}$ In ARDS patients with normally aerated lung regions at zero PEEP (focal ARDS, characterized by radiological attenuations predominating in the lower quadrants), the static PV curve depends both on the elastance/compliance of aerated lung regions and on the recruitment of poorly and non-aerated pulmonary areas. $^{7}$

Lung recruitment at a given airway pressure can be defined as the difference in lung volume between PV curves starting at different end-expiratory lung volumes corresponding to different PEEP. This method was validated against the reference computed tomography scan method, ${ }^{2}$ defining PEEP-induced alveolar recruitment as the amount of gas penetrating into poorly and non-aerated lung regions after PEEP. ${ }^{7}$ Two methods based on PV curves and providing automatic measurement of PEEP-induced alveolar recruitment have been implemented on mechanical ventilators and are actually commercially available: the PV recruitment module on the eXtend ventilator (Air Liquide Medical Systems, Antony, France) and the PEEP Inview module of the Engström Carestation (GE Healthcare, Madison, Wisconsin).

\section{Automatic Methods Available on Mechanical Ventilators and Based on Pressure-Volume Curves}

The PV recruitment module of the eXtend ventilator exactly reproduces the reference method proposed by Ranieri et al. ${ }^{5}$ The ventilator is equipped with a flow generator providing a constant flow ranging between 1 and $10 \mathrm{~L} / \mathrm{min}$. It has been shown that PV curves obtained in static conditions (gross syringe or end-inspiratory occlusion methods) are equivalent to PV curves obtained in quasi-static conditions (constant flows of 3 or $9 \mathrm{~L} / \mathrm{min}$ ), ${ }^{10}$ 
with a high reproducibility. ${ }^{11}$ Before starting the automatic measurement of PEEP-induced alveolar recruitment, the clinician has to select the different PEEP levels (a maximum of 5), the constant flow rate (between 1 and $10 \mathrm{~L} /$ $\min$ ), the maximum inflation pressure (up to $60 \mathrm{~cm} \mathrm{H}_{2} \mathrm{O}$ ), the maximum volume administration during each PV curve, and the time passed at the highest PEEP to optimize initial lung recruitment. The ventilator performs a descending PEEP trial, because there is evidence that it optimizes PEEP-induced alveolar recruitment. ${ }^{12,13}$ At each PEEP the following sequence is performed: 10 respiratory cycles, PV curve, 10 respiratory cycles, and PEEP releasing maneuver. At the end of the procedure, all PV curves are displayed on the screen, starting from the corresponding end-expiratory lung volume. Alveolar recruitment at any airway pressure can be measured by moving a cursor on the screen. The lower inflection pressure, the slope of the PV curve (lung recruitability), and the upper distending pressure can be measured on each PV curve using the mobile cursor.

The procedure requires an anesthetized and paralyzed patient, is easy to perform, lasts between 2 and 6 min, and has the limitations of the method itself. During the maneuver, each PV curve is the equivalent of a recruitment maneuver, whereas each PEEP releasing maneuver is equivalent to a derecruitment maneuver. As such, these maneuvers could markedly interact with PEEP-induced alveolar recruitment. ${ }^{14}$ However, a study specifically looking at the impact of the procedure on gas exchange did not find any related significant changes in $\mathrm{PaO}_{2}$ and $\mathrm{PaCO}_{2} \cdot{ }^{15}$ Compared to the computed tomography reference technique, the PV curve method provides an acceptable estimation of PEEPinduced alveolar recruitment. ${ }^{2}$

The PEEP Inview module of the Engström Carestation is an indirect application of the reference method proposed by Ranieri et al. ${ }^{5}$ Two original modifications have been introduced to allow measurement of alveolar recruitment in dynamic conditions of mechanical ventilation: static PV curves using constant flow are replaced by dynostatic PV curves obtained during tidal inflation. ${ }^{16}$ The PEEP releasing maneuver is replaced by the measurement of endexpiratory lung volume using the nitrogen wash-out/ wash-in technique. ${ }^{17,18}$ The ventilator is equipped with a sensor specifically designed for measuring intratracheal pressure. By excluding inspiratory resistance of the endotracheal tube, measuring intratracheal pressure makes possible the accurate measurement of dynostatic PV curves in dynamic conditions. ${ }^{19,20}$ For routine clinical use, a 2-mm intraluminal and air-filled catheter with end-hole should be positioned in the trachea, $2 \mathrm{~cm}$ beyond distal tip of the endotracheal tube, ${ }^{18}$ and left in place throughout the period of measurement. Before starting automatic measurement of PEEP-induced alveolar recruitment, the clinician selects the different PEEP levels at which alveolar recruitment will be measured (a maximum of 5) according to an ascending PEEP trial, the tidal volume, and the maximal inflation pressure (up to $100 \mathrm{~cm} \mathrm{H}_{2} \mathrm{O}$ ). The time passed at each PEEP to allow accurate measurement of end-expiratory lung volume using the nitrogen wash-out/ wash-in technique is automatically determined (between a few minutes and 3 hours). At the end of the procedure, numeric values of the different end-expiratory lung volumes and recruited volume (computed as the difference in volume between each dynostatic curve) are displayed on the screen.

The procedure does not require a paralyzed patient, but a good coordination between the patient and the ventilator, and is easy to perform. The procedure lasts much longer than the classical PV curve method because it requires steady state conditions (10 $\mathrm{min}$ at least) between the different PEEP levels, in order to ensure nitrogen washout/wash-in. ${ }^{18}$ It has the limitations of the dynostatic and nitrogen wash-out/wash-in methods. Measurement of intratracheal pressure may be associated with errors in pressuremeasurement(end-holevsside-holecatheters, intraluminal diameter, catheter obstruction by tracheal secretions). ${ }^{16}$ Presence of an intratracheal catheter increases endotracheal tube resistance, interferes with tracheal suctioning, and may be the source of tracheal injury. The accuracy of nitrogen wash-out/wash-in decreases with $\mathrm{FIO}_{2}$ $>70 \%$ and is very sensitive to microleaks in ventilatory circuits and heterogeneity of regional gas distribution, frequently observed in ARDS. ${ }^{16}$ Until now, the technique has not been evaluated against the computed tomography reference method or compared to the quasi-static PV curves method.

\section{Methods Based on Transthoracic Lung Ultrasound}

As stated by the International Consensus Conference on Lung Ultrasound, there is strong evidence that "lung ultrasound is able to monitor aeration changes and the effect of therapy in patients with acute pulmonary edema, ARDS, community-acquired pneumonia, and ventilator-associated pneumonia." ${ }^{21}$ Bedside transthoracic ultrasound is based on the examination of 12 regions of interest: upper and lower parts of anterior, lateral, and posterior right and left chest walls. Following any therapy aimed at improving lung aeration, each region of interest may vary between 4 conditions: normal aeration (lung sliding and horizontal A lines), moderate loss of lung aeration related to interstitial syndrome (multiple spaced vertical B lines), severe loss of lung aeration related to alveolar-interstitial syndrome (coalescent vertical B lines), and complete loss of lung aeration caused by alveolar consolidation or atelectasis. $^{22}$ By adding changes in ultrasound pattern detected in each region of interest, ultrasound scores or re-aeration have been proposed and correlated to reference methods 


\section{Measurement of Alveolar Recruitment at the Bedside}

for quantifying lung aeration changes (computed tomography, extravascular lung water, pulmonary wedge pressure, PV curves, lung lavage for alveolar proteinosis). ${ }^{4,22-27}$ Bedside transthoracic lung ultrasound has been demonstrated to be accurate for assessing re-aeration following fluid depletion in patients with hemodynamic pulmonary edema, PEEP-induced alveolar recruitment in ARDS, ${ }^{4}$ lung re-aeration resulting from efficient antimicrobial therapy in patients with community-acquired ${ }^{28}$ or ventilator-associated pneumonia, ${ }^{22}$ and derecruitment observed at the early phase of acute lung injury ${ }^{29}$ or during a spontaneous breathing trial. ${ }^{30}$ Two different lung ultrasound scores have been proposed to assess lung recruitment and derecruitment induced by various treatments and lung diseases.

In patients with interstitial and pulmonary edema, the ultrasound score is based on the number of vertical B lines detected in anterior and lateral parts of the chest wall (8 regions of interest). ${ }^{31,32}$ Four intercostal spaces are examined in the right side, and 3 in the left side, and the presence of coalescent vertical B lines is quoted $10 \mathrm{~B}$ lines. The lung ultrasound score is significantly correlated to extravascular lung water measured by gravimetry, ${ }^{33}$ the indicator dilution method, ${ }^{23}$ or computed tomography. ${ }^{26}$

In patients with ARDS and/or pneumonia, consolidated lung areas predominating in dependent lung regions coexist with interstitial and alveolar edema present in anterior and lateral lung regions. Therefore a lung ultrasound score aimed at assessing lung recruitment and derecruitment should include dependent lung regions, which are frequently consolidated. Lung ultrasound examination is performed on 12 regions of interest, including upper and lower posterior regions, which require lateral positioning for appropriate evaluation. In each region examined, changes in ultrasound pattern following therapeutic intervention is detected and numbers are attributed as follows: from multiple B lines to normal, from coalescent B lines to multiple $\mathrm{B}$ lines, and from consolidation to coalescent B lines, 1 point; from coalescent $\mathrm{B}$ lines to normal, or from consolidation to multiple B lines, 3 points; from consolidation to normal, 5 points. The mean lung ultrasound score of re-aeration is then calculated for the 12 regions examined. A statistically significant correlation was found between this score and CT assessment of lung re-aeration following

Dr Rouby and Dr Lu have disclosed a relationship with Air Liquide Medical Systems. The other authors have disclosed no conflicts of interest.

Correspondence: Jean-Jacques Rouby MD PhD, Réanimation Polyvalente Pierre Viars, Department of Anesthesiology and Critical Care, PitiéSalpétrière Hospital, 47-83 Boulevard de l'Hôpital 75013, Paris, France. E-mail: jjrouby@invivo.edu.

DOI: $10.4187 /$ respcare. 02363 efficient antimicrobial therapy in patients with ventilatorassociated pneumonia. ${ }^{22}$ Following PEEP administration to patients with ARDS, a significant correlation was found between alveolar recruitment measured by the PV curve method and the ultrasound re-aeration score. ${ }^{4}$

Bedside monitoring of alveolar recruitment (or derecruitment) has entered the clinical area and should improve in the close future the ventilatory management of patients with ARDS. Because it is noninvasive and easily repeatable, bedside transthoracic lung ultrasound appears as the most promising semi-quantitative technique. At the early phase of ARDS the static PV curve method using constant flow is the most accurate quantitative tool to measure lung recruitment but requires a paralyzed patient. Methods based on dynostatic PV curves and direct measurement of end-expiratory lung volume by the nitrogen wash-out/wash-in technique are promising because they are less invasive, but they require validation before being recommended for clinical use. Even if methods assessing changes in radiological density on bedside chest radiographies seem to be far from clinical routine application, ${ }^{1}$ they could be an attractive alternative if density measurements could be automatic and independent of the radiologist.

Jean-Jacques Rouby MD PhD Charlotte Arbelot MD Hélène Brisson MD Qin Lu MD PhD Multidisciplinary ICU Department of Anesthesiology and

Critical Care Medicine

La Pitié-Salpêtrière Hospital Assistance Publique Hôpitaux de Paris University School of Medicine Pierre and

Marie Curie

Paris, France

Belaïd Bouhemad MD PhD

Surgical ICU

Department of Anesthesiology and

Critical Care Medicine

Groupe Hospitalier Paris-Saint Joseph

Assistance Publique Hôpitaux de Paris University School of Medicine René Descartes

Paris, France

\section{REFERENCES}

1. Wallet F, Delannoy B, Haquin A, Debord S, Leray V, Bourdin G, et al. Evaluation of recruited lung volume at inspiratory plateau pressure with PEEP using bedside digital chest $\mathrm{x}$-ray in patients with acute lung injury/ARDS. Respir Care 2013;58(3):416-423.

2. Lu Q, Constantin JM, Nieszkowska A, Elman M, Vieira S, Rouby JJ. Measurement of alveolar derecruitment in patients with acute lung 


\section{Measurement of Alveolar Recruitment at the Bedside}

injury: Computerized tomography versus pressure-volume curve. Crit Care 2006;10(3):R95. DOI: 10.1186/cc4956.

3. Dellamonica J, Lerolle N, Sargentini C, Beduneau G, Di Marco F, Mercat A, et al. PEEP-induced changes in lung volume in acute respiratory distress syndrome: two methods to estimate alveolar recruitment. Int Care Med 2011;37(10):1595-1604.

4. Bouhemad B, Brisson H, Le Guen M, Arbelot C, Lu Q, Rouby JJ. Bedside ultrasound assessment of positive-end expiratory pressureinduced lung recruitment. Am J Respir Crit Care Med 2011;183(3): 341-347.

5. Ranieri VM, Eissa NT, Corbeil C, Chassé M, Braidy J, Matar, et al. Effects of positive end-expiratory pressure on alveolar recruitment and gas exchange in patients with the adult respiratory distress syndrome. Am Rev Respir Dis 1991;144(9):544-551.

6. Hickling KG. The pressure-volume curve is greatly modified by recruitment. A mathematical model of ARDS lungs. Am J Respir Crit Care Med 1998;158(1):194-202.

7. Rouby JJ, Lu Q, Vieira S. Pressure/volume curves and lung computed tomography in acute respiratory distress syndrome. Eur Respir J 2003;42(Suppl):27S-36S

8. Malbouisson L, Muller JC, Constantin JM, Lu Q, Puybasset L, Rouby JJ; CT Scan ARDS Study Group. Computed tomography assessment of positive end-expiratory pressure-induced alveolar recruitment in patients with acute respiratory distress syndrome. Am J Respir Crit Care Med 2001;163(6):1444-1450.

9. Rouby JJ, Puybasset L, Nieszkowszka A, Lu Q. Acute respiratory distress syndrome: lessons from computed tomography of the whole lung. Crit Care Med 2003;31(4 Suppl):S285-S295.

10. Lu Q, Vieira SRR, Richecoeur J, Puybasset L, Kalfon P, Coriat P, et al. A simple automated method for measuring pressure-volume curves during mechanical ventilation. Am J Respir Crit Care Med 1999;159(1):275-282.

11. Mehta S, Steward TE, MacDonald R, Hallet D, Banayan D, Lapinsky A, et al. Temporal changes, reproducibility and interobserver variability in pressure-volume curves in adults with acute lung injury and acute respiratory distress syndrome. Crit Care Med 2003;31(6): 2118-2125

12. Rouby JJ. Optimizing lung aeration in positive end-expiratory pressure. Am J Respir Crit Care Med 2004;17(10):1039-1040.

13. Albaiceta GM, Taboada F, Parra D, Luyando LH, Calvo J, Menendez L, et al. Tomographic study of the inflection points of the pressure-volume curve in acute lung injury. Am J Respir Crit Care Med 2004;170(10):1066-1072.

14. Brochard L. What is a pressure-volume curve? Crit Care 2006;10(7): R156. DOI: $10.1186 / \mathrm{cc} 5002$

15. Roche A, Forel JM, Demory D, Arnal JL, Donati S, Gainnier M, et al. Generation of a single pulmonary pressure-volume curve does not affect durably oxygenation in ARDS patients. Crit Care 2006; 10(8):R85. DOI: $10.1186 / \mathrm{cc} 5002$.

16. Karason S, Sondergaard S, Lundin S, Wiklund J, Stenqvist O. Evaluation of pressure-volume loops based on intratracheal pressure measurements during dynamic conditions. Acta Anaesthesiol Scand 2000; 44(5):571-577.

17. Olegard C, Sondergaard S, Houltz E, Lundin S, Stenqvist O. Estimation of functional residual capacity at the bedside using standard monitoring equipment: a modified nitrogen washout/washin technique requiring a small change of the inspired oxygen fraction. Anesth Analg 2005;101(1):206-212.

18. Dellamonica J, Lerolle N, Sargentini C, Beduneau B, Di Marco F, Mercat A, et al. Accuracy and precision of end-expiratory lung- volume measurements by automated nitrogen washout/washin technique in patients with acute respiratory distress syndrome. Crit Care 2011;15(6):R294. DOI: 10.1186/cc10587.

19. Karason S, Sondergaard S, Lundin S, Wiklund J, Stenqvist O. A new method for non-invasive, free- manoeuver determination of "static" pressure-volume curves during dynamic/therapeutic mechanical ventilation. Acta Anaesthesiol Scand 2000;44(5):578-585.

20. Karason S, Sondergaard S, Lundin S, Stenqvist O. Continuous online measurements of respiratory system, lung and chest wall mechanics during mechanical ventilation. Int Care Med 2001;37(8): 132-1339.

21. Volpicelli G, Elbarbary M, Blaivas M, Lichtenstein DA, Mathis G, Kirkpatrick AW, et al; International Liaison Committee on Lung Ultrasound (ILC-LUS). International evidence-based recommendations for point-of-care lung ultrasound. Int Care Med 2012;38(4): 577-591.

22. Bouhemad B, Liu ZH, Arbelot C, Zhang M, Ferarri F, Le-Guen M, et al. Ultrasound assessment of antibiotic-induced pulmonary reaeration in ventilator-associated pneumonia. Crit Care Med 2010;38(1): 84-92.

23. Agricola E, Bove T, Oppizzi M, Marino G, Zangrillo A, Margonato A, Picano E. "Ultrasound comet-tail images": a marker of pulmonary edema: a comparative study with wedge pressure and extravascular lung water. Chest 2005;127(5):1690-1695.

24. Agricola E, Picano E, Oppizzi M, Pisani M, Meris A, Fragasso G, Margonato A. Assessment of stress-induced pulmonary interstitial edema by chest ultrasound during exercise echocardiography and its correlation with left ventricular function. J Am Soc Echocardiogr 2006;19(4):457-463.

25. Via G, Lichtenstein D, Mojoli F, Rodi G, Neri L, Storti E, et al. Whole lung lavage: a unique model for ultrasound assessment of lung aeration changes. Int Care Med 2010;36(6):999-1007.

26. Baldi G, Gargani L, Abramo A, D'Errico L, Caramella D, Picano E, et al. Lung water assessment by lung ultrasonography in intensive care: a pilot study. Int Care Med 2013;39(1):74-84.

27. Picano E, Frassi F, Agricola E, Gligorova S, Gargani L, Mottola G. Ultrasound lung comets: a clinical sign of extravascular lung water. J Am Soc Echocardiogr 2006;19(3):356-363.

28. Reissig A, Copetti R, Mathis G, Mempel C, Schuler A, Zechner C, et al. Lung ultrasound in the diagnosis and follow-up of communityacquired pneumonia: a prospective multicenter accuracy study. Chest 2012;142(4):965-972.

29. Gargani L, Lionetti V, Di Cristofano C, Bevilacqua G, Recchia FA, Picano E. Early detection of acute lung injury uncoupled to hypoxemia in pigs using ultrasound lung comets. Crit Care Med 2007; 35(12):2769-2774

30. Soummer A, Perbet S, Brisson H, Arbelot C, Constantin JM, Lu Q, et al; Lung Ultrasound Study Group. Ultrasound assessment of lung aeration loss during a successful weaning trial predicts postextubation distress. Crit Care Med 2012;40(7):2064-2072.

31. Jambrik Z, Monti S, Coppola V, Agricola E, Mottola G, Miniati M, et al. Usefulness of ultrasound lung comets as a nonradiologic sign of extravascular lung water. Am J Cardiol 2004;93(10):1264-1270

32. Gargani L. Lung ultrasound: a new tool for the cardiologist. Cardiovasc Ultrasound 2011;(9):6.

33. Jambrik Z, Gargani L, Adamicza A, Kaszaki J, Varga A, Forster T, et al. B-lines quantify the lung water content: a lung ultrasound versus lung gravimetry study in acute lung injury. Ultrasound Med Biol 2010;36(12):2004-2010. 